\title{
EFFECTS OF FRAGILITY FRACTURE INTEGRATED REHABILITATION MANAGEMENT HIP FRACTURE IN PAKISTANI OLDER ADULTS
}

1. Assistant Professor Riphah College of Rehabilitation \& Allied Sciences, Riphah International University, Islamabad Pakistan

2. Professor. Isra Institute of Rehabilitation Sciences, Islamabad. Pakistan

Lecturer/Doctor of Physical Therapy. Helping

Hand institute of Rehabiltation Hand Institute of Rehabilitation sciences. Mansehra Pakistan

\section{Correspondence}

Anam Aftab

Assistant Professor/In-charge Research (MSPT)

Riphah College of Rehabilitation \& Allied Sciences,

Riphah International University, Islamabad Pakistan E-mail:anam.aftab@rihah.edu.pk

Received on: 24-11-2020 Revision on: 10-06-2021

Published on: 30-06-2021

Citation; Aftab A, Habibullah S, Bhutta NI. Effects of fragility fracture integrated rehabilitation management on hip fracture in pakistani older adults. T Rehabili. J. 2021:05(01); 192-197 soi: 21-2017/re-trivol05iss01p192

doi: https://doi.org/10.52567/tri.v5i01.55
Anam Aftab: Conception, writing. Revised and accountable for all aspects

Shaista Habibullah ${ }^{2}$ : Revised and accountable for all aspects

Nimra llyas Bhutta ${ }^{3}$ Analysis \& interpretation of data, Revised and accountable for all aspects

\section{ABSTRACT}

Objective: to evaluate the effectiveness of fragility Fracture Integrated Rehabilitation Management (FIRM) on older adults of Pakistan after hip fracture surgery. Methodology: A one-group pretest-posttest design was conducted at the National Institute of Rehabilitation Medicine (NIRM), Islamabad from March 2020 to September 2020. A total of $n=11$ participants with age above 55 years, both male and female, with confirming diagnosed cases of hip fracture were included. Every participants received 10 sessions of Fragility Fracture integrated Rehabilitation Management (FIRM) program in two weeks. Data was collected at baseline at $2^{\text {nd }}$ day and after 10th session on 15th day, through the KOVAL scale to assess the walking ability, the Functional Ambulatory Category (FAC) to assess the level of independence, the modified Barthel index (MBI) for activities of daily living (ADLs), and quality of life (QOL) was assessed by EQ-5D. Data were analysed by using SPSS Version 21. Result: the mean age $76.45 \pm 9.32$, of which $n=6(54.6 \%)$ were males and $n=5(45.4 \%)$ were females. after 10th sessions the QOL on EQ-5D, ambulation on FAC and KOVAL scale and the $\mathrm{ADL}$ on $\mathrm{MBI}$ were significantly improved $(p<0.05)$ with large effect size, except for the anxiety domain of EQ5D and subdomains of MBI; Personal hygiene, feeding, Bowel control, bladder control, Wheelchair, and Chair \& bed transfer showed no significant change $(p>0.05)$. Conclusion: FIRM care is found to be effective in improving the walking ability, functional status, ADLs and quality of life in older adults following a hip fracture surgery.

Keywords: Activity of daily living (ADLS), fragility fracture, physical therapy, rehabilitation

\section{INTRODUCTION}

A fragility fracture is a commonly occurring condition resulting from low energy mechanical forces among the old aged population specifically above 50 years. Among three major sites for fragility fracture, the hip fragile fracture is the most common condition in geriatrics. ${ }^{1,2}$. Hip fractures are major fractures and nearly always require hospitalization, which is fatal in $20 \%$ of cases and cause of permanent disability in $50 \%$ of these affected cases, whereas only $30 \%$ of these cases had shown full recovery. ${ }^{3}$. By 2050 , it is suspected that there will be 6.26 million hip fractures worldwide of which approximately $50 \%$ will occur in Asia, but there is only a small number of reports on the incidence of hip fractures in the Asian population. . Gender-wise, women are more affected than men, as they have a higher incidence of osteoporosis due to increased bone loss after menopause in women. ${ }^{5}$ Most of the fractures are due to fall, whereas the hip fractures occur both outside or inside the house, while the occurrence of fragility fractures are somewhat more commonly reported outside the house. ${ }^{6}$. Hip fractures have been reported to be the costliest of all the fragility fractures, such fractures can prompt serious morbidity, reduce the quality of life, and are associated with high mortality rates. ${ }^{7,8}$
There are several therapies and treatments available for the prevention and rehabilitation of fragility fractures in people who are thought to be at risk or to prevent further fractures in those who have already had one or more fragility fractures. However, identifying who will benefit from preventative and rehabilitative treatment is imprecise. ${ }^{9}$ The conventionally used interventions include minimally invasive surgery, anesthetics, and conventional radiography which are commonly being practiced for healing and fixing fragile hip fractures. $^{10-12}$ It is recognized that good multidisciplinary teamwork and care are essential for fragility fracture patients, and aspects of interventions undertaken throughout the care pathway need a more structured assessment through randomized controlled trials. ${ }^{13}$ As healing of occurring fractures is not enough, a comprehensive rehabilitation program is required to rehabilitate and prevent further fracture in geriatrics and to improve the functional status of patients after surgery recovery. ${ }^{14}$. The Importance and effectiveness of a comprehensive rehabilitation program after fragile hip fracture in old aged population is explained well by Abraham et al. in their recently conducted study. ${ }^{15}$ For this purpose comprehensive rehabilitation care is integrated internationally for geriatric care after fragile hip 
fracture known as Fragility Fracture integrated Rehabilitation Management (FIRM), requiring a multidisciplinary team including orthopaedic doctors, physiotherapists, occupational therapists and nurses geriatricians. This protocol was incorporated by a Korean Fragility Fracture Rehabilitation study group, with a motive to decrease the level of dependency, and risk of falls, with detailed discharging plans. This protocol consists of multidisciplinary team work-integrated sessions with 10 days of physiotherapy and four days of occupational therapy post-surgical recovery of fracture, gradually increasing according to patients functional status. ${ }^{16}$

Numerous studies regarding the fragility of integrated rehabilitation have shown benefits for functional outcomes in patients with fragility hip fractures. The use of fragility integrated rehabilitation leads to improvements in functional outcomes, mobility, cognition, and overall healthrelated quality of a life. ${ }^{16-21}$ As per authors' knowledge, this intervention has not been practiced and tested as well as not any other standard rehabilitation protocol on hip fracture among the older adults of Pakistan. Therefore, this study is aimed to evaluate the effectiveness of
FIRM on the Pakistani adult population after hip fragile fracture surgery.

\begin{tabular}{lll}
\hline \hline METHODOLOGY & \\
\hline \hline A one-group pretest-posttest study
\end{tabular}

(NCT04760756) was conducted for a duration of 6 months from March 2020 to September 2020, after approval from the Deputy Director Admin (No,F.170/2019-Admin/NIRM) of National Institute of Rehabilitation Medicine (NIRM) Islamabad. The $\mathrm{n}=17$ patients were evaluated for eligibility and $\mathrm{n}=11$ older adults with age above 55 years, diagnosed with femoral neck, sub-trochanteric or intertrochanteric fracture and underwent hip fracture management including any of bipolar hemiarthroplasty, total hip replacement arthroplasty, reduction, or internal fixation, were included in the study.(Figure 1)

The participants who declined to participate in the study, had undergone surgery for a cause other than hip fracture; (infection, arthritis, loosening, avascular necrosis, femoral shaft fracture, acetabular fracture, peri-prosthetic fracture or pathological fracture by tumor or had isolated fracture of the greater or lesser tuberosity, or multiple fractures), or having a history of repeated surgery for hip fracture were excluded.

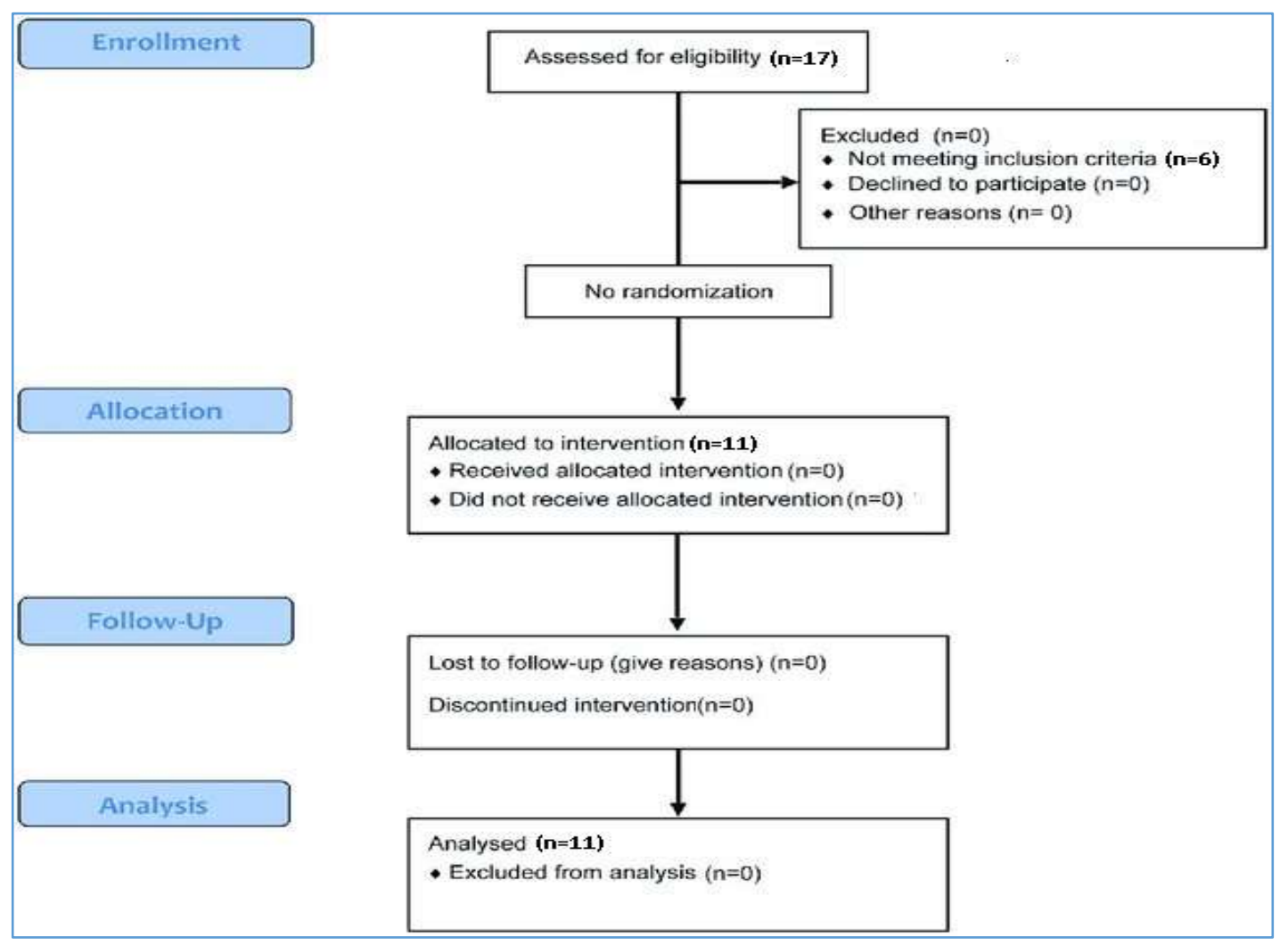

Figure 1: Study Flowchart 
Each participant was admitted for 15 days for the comprehensive rehabilitation program. The FIRM program was provided by a multidisciplinary team which included; a Physician, occupational therapist, Physical therapists, social worker, clinical nurse, and a nutritionist. The aim of adding occupational and physical therapies was to improve the activities of daily living and mobility. A total of 10 sessions of Physical therapy were provided for about a duration of 40 mins session/day (FIRM\#1-10); which included strengthening, aerobics, functional, weight-bearing, and gait-training exercises, whereas, four sessions of occupational therapy were provided onwards $4^{\text {th }}$ session of physical therapy and every alternate day (FIRM\#4,6,8, 10 ) which included training for the use of adaptive equipment, self-care, sit to stand, bed mobility, transfer, self-dressing, and ADLs. Comprehensive patient education was also part of this rehabilitative program. $^{21}$

The demographic data (age, gender, and BMI) was obtained from each participant. Data was collected at baseline on $2^{\text {nd }}$ day and after $10^{\text {th }}$ session on $15^{\text {th }}$ day, through the KOVAL scale to assess the walking ability $^{13}$, the Functional Ambulatory Category
$(F A C)^{13}$ to assess the level of independence, the modified Barthel index (MBI) ${ }^{22}$ for activities of daily living (ADLs), and quality of life (QoL) was assessed by $E Q-5 D^{23}$

The data presented in th form of frequency $(n)$, percentages, median and Interquartile range (IQR), $p$-value and effect size $(r)$. As variables were in ordinal scale and total score was not normally distributed, so the non-parametric Wilcoxon Sign Ranked Test was used for pre-post analysis. The level of significance was set at $p<0.05$ and correlation coefficient $(r)$ was used to determine the effect size.

\begin{tabular}{l} 
RESULTS \\
\hline A total of $n=11$ participated in the study. The mean \\
age of the study participants was $76.45 \pm 9.32$. \\
Regarding gender distribution in the study, a total \\
of $n=6$ males and $n=5$ females participated in the \\
study. In which the height of the participants was \\
$159.02 \pm 8.07$ and weight was $57.56 \pm 7.22$. The \\
average BMI of the participant was $22.99 \pm 3.95$. \\
(Figure 2)
\end{tabular}
(Figure 2)

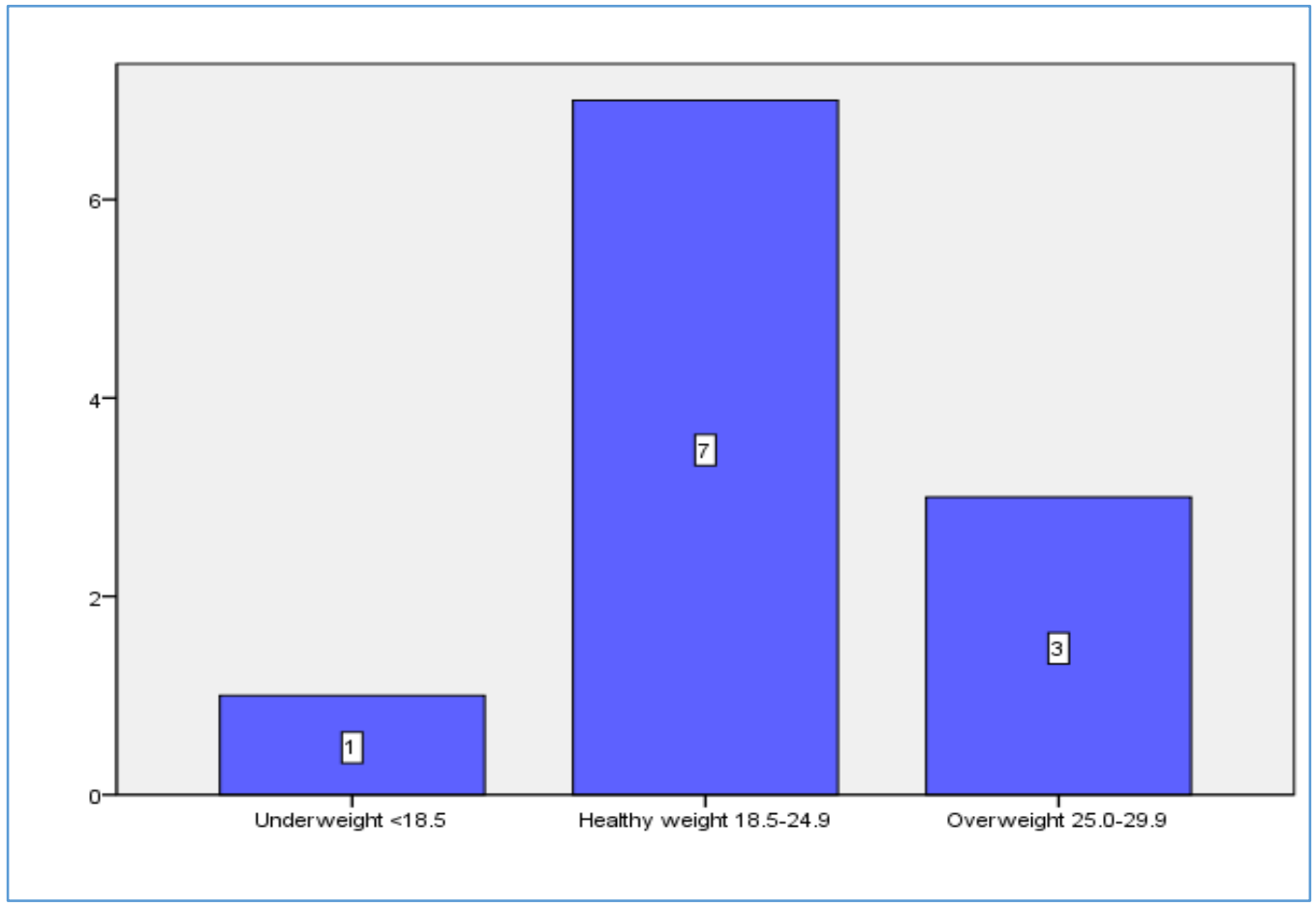

Figure 2: Frequency distribution of study participant

The FIRM protocol was given to all participants and the data was analyzed after 10 days. The analysis showed that quality of life on EQ5D significantly improved with large effect size $\{0.316(0.201)$ ver. 0.573 (0.107), $p=0.003, r=-0.88\}$. All domains of EQ5D also showed significant improvement 
$(p<0.05)$ with large effect size, except in anxiety $(p>0.05)$. The ambulation also showed significant improvement with large effects size measured on Functional ambulatory category scale $\{1(1)$ ver. $3(1), p=0.010, r=-2.58\}$ and KOVAL $\{6(1)$ ver. 6(0), $p=0.025, r=-2.23\}$ respectively.

The activities of daily living on modified Barthel index (MBI) showed a significant improvement $\{61(17.750)$ ver. $78(15.500) p=0.005, r=-0.84\}$. While considering the subdomains of $\mathrm{MBI}$, the following domains significantly improved with large effect sizes such as bathing self, Toilet, stair climbing, dressing, and ambulation. While remaining domains, no significant change $(p>0.05)$ was observed including Personal hygiene, feeding, Bowel control, bladder control, Wheelchair, and Chair \& bed transfer.

Table 1: Pre and post-analysis of Study variables

\begin{tabular}{|c|c|c|c|c|c|c|c|c|}
\hline & & \multicolumn{2}{|c|}{ Pre } & \multicolumn{2}{|c|}{ Post } & \multirow{2}{*}{$Z$} & \multirow{2}{*}{$p$-value } & \multirow{2}{*}{$r$} \\
\hline & & Median & IQR & Median & IQR & & & \\
\hline \multirow{6}{*}{ EQ5D } & Exercise & 4 & 2 & 2 & 2 & -2.83 & $0.005 * *$ & -0.85 \\
\hline & Self-care & 4 & 0 & 3 & 2 & -2.58 & $0.010^{*}$ & -0.78 \\
\hline & ADLs & 5 & 1 & 4 & 0 & -2.27 & $0.023^{*}$ & -0.68 \\
\hline & Pain & 4 & 1 & 2 & 1 & -3.20 & $0.001^{* *}$ & -0.96 \\
\hline & Anxiety & 2 & 1 & 1 & 1 & -1.34 & 0.18 & -0.67 \\
\hline & Total & 0.316 & 0.201 & 0.573 & 0.107 & -2.93 & $0.003 * *$ & -0.88 \\
\hline FAC & - & 1 & 1 & 3 & 1 & -2.58 & $0.010^{*}$ & -2.58 \\
\hline KOVAL & - & 6 & 1 & 6 & 0 & -2.23 & $0.025^{*}$ & -2.23 \\
\hline \multirow{12}{*}{$\mathrm{MBI}$} & Personal hygiene & 4 & 1 & 4 & 1 & -1.00 & 0.317 & -1 \\
\hline & Bathing self & 1 & 3 & 3 & 3 & -2.12 & $0.034^{*}$ & -10.55 \\
\hline & feeding & 10 & 2 & 10 & 0 & -1.00 & 0.317 & -1 \\
\hline & Toilet & 3 & 3 & 5 & 6 & -2.22 & $0.026^{*}$ & -2.22 \\
\hline & Stair climbing & 0 & 0 & 5 & 8 & -2.45 & $0.014^{*}$ & -2.45 \\
\hline & Dressing & 5 & 3 & 5 & 3 & -2.41 & $0.016 *$ & -0.80 \\
\hline & Bowel control & 10 & 2 & 10 & 2 & -1.00 & 0.317 & -0.5 \\
\hline & Bladder control & 10 & 0 & 10 & 0 & -1.00 & 0.317 & -0.33 \\
\hline & Ambulation & 3 & 8 & 12 & 4 & -2.55 & $0.011^{*}$ & -0.77 \\
\hline & Wheel chair & 0 & 0 & 0 & 0 & 0.00 & 1 & 0 \\
\hline & Chair \& bed transfer & 12 & 9 & 12 & 4 & -1.84 & 0.066 & -0.92 \\
\hline & Total MBI & 61 & 17.75 & 78 & 15.5 & -2.80 & $0.005^{* *}$ & -0.84 \\
\hline
\end{tabular}

Significance Level: $p<0.05^{*}, p<0.01^{* *}, p<0.001^{* * *}$.

The correlation coefficient ( $r$ ) for effect size

\section{DISCUSSION}

This pre-post study was aimed to evaluate the effectiveness of Fracture integrated rehabilitation Management (FIRM) care on Pakistani older adults after hip fracture surgery. The results highlighted that after 10 sessions of FIRM care there was a significant improvement in the functional and mobility status of geriatric patients. And there exists a significant change in the KOVAL, FAC, MBI, and quality of life EQ-5D when assessed the mean difference of pre and post scores in a patient with a fragile hip fracture.

The present study statistics of the within-group analysis showed that the walking ability was significantly improved after 15 days of hip fracture fixation surgery. These findings are supported by several studies conducted for geriatric hip fracture rehabilitation. $^{21,24,25}$ Lee et al. reported in their recently published study that when measured on the KOVAL scale the ADLs, physical and walking abilities are improved in elderly postoperative patients who are enrolled in the FIRM care program. ${ }^{16}$ These findings are also evident in a study conducted on Korean adult population that FIRM care improves walking ability with crutches and walkers in elderly patients receiving rehabilitation post-hip fracture surgery. ${ }^{18}$ A metaanalysis that evaluated the hip fracture rehabilitation effect on the geriatric population reported that those rehabilitation programs which are systematically planned by the geriatric interdisciplinary team approach are more effective in improving physical ability, walking abilities, and 
ADLs in the elderly population than any of the conventional care. $^{25}$

The present study highlighted that the functional ambulatory category was seemed to be improved when analyzed for within-group change from baseline to $10^{\text {th }}$ session at $15^{\text {th }}$ day postoperatively. This indicates that after surgical correction of fragile hip fracture, the FIRM is significantly effective in enhancing the functional ambulation of elderly patients. A study conducted on the effectiveness of FIRM on elderly patients with and without Sarcopenia reported that that FIRM care is clinically useful in enhancing the functional gain in ambulatory functions, quality of life walking ability, and balance. ${ }^{20}$ Similar statistics are reported by another rehabilitative study conducted on the evaluation of FIRM care on functional outcomes improvement and predictor for independent ambulation, reported that FIRM is an intensive, standardized, comprehensive rehabilitative program, which had a positive short term, as well as long term effects in the elderly population suffering a hip fracture. This intensive interdisciplinary team approach is thought to be satisfactorily subtle for enhancing the functional outcomes in Sarcopenia patients with hip fracture and is a prognostic predictor for independent ambulation. ${ }^{19}$

The quality of life for activities of daily living was measured through the EUR-Q5 dimensions questionnaire, which showed significant improvement in four dimensions except for the anxiety, which was insignificant. In a study conducted among the Korean community regarding the quality of life in elderly patients with hip fracture, reported that level of pain, discomfort, mobility, anxiety, and usual activities were significantly improved in patients receiving FIRM care when assessed $10^{\text {th }}$ days postoperatively. ${ }^{21}$ These findings are also evident by research, which reported that the interdisciplinary team rehabilitative care is effective in improving the quality of life and activities of daily living by enhancing physical functioning and reducing pain and discomfort level (26) whereas, another study supports these finding that FIRM protocol is effective in improving the quality of life, range of motion and activities of daily living by reducing pain and discomfort level in such patients. ${ }^{18}$
This study statistics showed that self-bathing, toilet training, stair climbing, self-dressing, ambulation were significantly improved except for personal hygiene, self-feeding, wheelchair, transfer between chair and bed, bowel, and bladder control. A recently published pre-test-post-test clinical trial evaluated the effect of FIRM care on the quality of life of the geriatric population suffering hip fragility fracture, assessed through modified Barthel index, and reported that the post-intervention analysis showed improvement in the quality of life after $10^{\text {th }}$-day session postoperatively, all activities were significantly improved. This provides a piece of strong evidence for our study results. ${ }^{21}$ As reported in the previous studies that reducing the level of rehabilitation is associated with decreased physical functioning after hip fracture, thus dependency increases and the quality of life gets worst. ${ }^{27,28}$ Although, in fragile geriatric population ambulation and ADLs recovery is a key factor in coping up with and returning to the daily life activities, therefore comprehensive rehabilitation care can help cope up and returning to the activities of daily living by enhancing the quality of life and mobility in such patients. $^{25,29}$

The current study had low sample, single centred without comparative group as well as long term effects are missing. These confounding factors limit the generalizability of the study. As compared to developed countries, imitated access to rehabilitation services and non-compliance with home plan may impede the progress. So long term effect should also be observed as after discharge at least for 6 months

\section{CONCLUSION}

Fracture integrated rehabilitation Management (FIRM) program is effective to improve level of mobility/ambulation, activities of daily livings (ADLs) and quality of life among older adults with hip fracture.

\section{REFERENCES}

1. Fortington LV, Rommers GM, Geertzen JH, Postema K, Dijkstra PU. Mobility in elderly people with a lower limb amputation: a systematic review. J Am Med Dir Assoc. 2012 May;13(4):319-25. doi: 10.1016/j.jamda.2010.12.097. Epub 2011 Mar 17. PMID: 21450239.Penn-Barwell J. Outcomes in lower limb 
amputation following trauma: a systematic review and meta-analysis. Injury 2011; 42(12): 1474-1479.

2. Rathore FA, Ayaz SB, Mansoor SN, Qureshi AR, Fahim M. Demographics of Lower Limb Amputations in the Pakistan Military: A Single Center, Three-Year Prospective Survey. Cureus. 2016 Apr 11;8(4):e566. doi: 10.7759/cureus.566. PMID: 27186448; PMCID: PMC4866834

3. Zidarov D, Swaine B, Gauthier-Gagnon C. Quality of life of persons with lower-limb amputation during rehabilitation and at 3-month follow-up. Arch Phys Med Rehabil. 2009 Apr;90(4):634-45. doi: 10.1016/j.apmr.2008.11.003. PMID: 19345780.

4. Khan A, Junaid N. Prevalence of diabetic foot syndrome amongst population with type 2 diabetes in Pakistan in primary care settings. J Pak Med Assoc. 2017 Dec;67(12):1818-1824. PMID: 29256523.

5. Molina CS, Faulk J. Lower Extremity Amputation. 2020 Sep 21. In: StatPearls [Internet]. Treasure Island (FL): StatPearls Publishing; 2020 Jan-. PMID: 31536201.

6. Amir Shahzad, Liaqat Ali Malik, Hamid Hussain, Suhail Karim Soomro, CAUSES OF AMPUTATION IN PAKISTANI POPULATION. Int. J Rehabili Sci 2016:5:54-57.

7. Humail SM, Ilyas S, Baqai FU: Diabetic foot: major cause of lower Limb amputations. J SurgPak. 2004, 9:19-21.

8. Razzaq S, Mansoor SN, Rathore FA, Akhter N, Yasmeen R: Functional outcomes following lower extremity amputation at the armed forces institute of rehabilitation medicine using lower extremity functional scale. Pak Armed Forces Med J. 2013, 63:52-56.

9. Mehr DR, Binder EF, Kruse RL, Zweig SC, Madsen R, Popejoy L, D'Agostino RB. Predicting mortality in nursing home residents with lower respiratory tract infection: The Missouri LRI Study. JAMA. 2001 Nov 21;286(19):2427-36. doi: 10.1001/jama.286.19.2427. PMID: 11712938.

10. Manton KG. A longitudinal study of functional change and mortality in the United States. J Gerontol. 1988 Sep;43(5):S153-61. doi: 10.1093/geronj/43.5.s153. PMID:

11. 2971088.Sinha $R$, van den Heuvel WJ, Arokiasamy P. Factors affecting quality of life in lower limb amputees. Prosthet Orthot Int. 2011 Mar;35(1):90-6. doi: 10.1177/0309364610397087. PMID: 21515894.

12. Davie-Smith F, Coulter E, Kennon B, Wyke S, Paul L. Factors influencing quality of life following lower limb amputation for peripheral arterial occlusive disease: A systematic review of the literature. Prosthet Orthot Int. 2017 Dec;41(6):537-547. doi: 10.1177/0309364617690394. Epub 2017 Feb 2. PMID: 28147898.

13. Wurdeman SR, Stevens PM, Campbell JH. Response to: Comments regarding: Mobility Analysis of AmpuTees (MAAT I): quality of life and satisfaction are strongly related to mobility for patients with a lower limb prosthesis by Wurdeman et al. Prosthet Orthot Int 2018 Aug;42(4):461-2.

14. Gómez, M. M. N., Gutiérrez, R. M. V., Castellanos, S. A. O., Vergara, M. P., \& Pradilla, Y. K. R. (2010). Psychological well-being and quality of life in patients treated for thyroid cancer after surgery. Terapia Psicológica, 28(1), 69-84.

15. Rantz MJ, Popejoy L, Zwygart-Stauffacher M, Wipke-Tevis D, Grando VT. Minimum Data Set and Resident Assessment Instrument. Can using standardized assessment improve clinical practice and outcomes of care? J Gerontol Nurs 1999; 25: 35-43

16. Zidarov D, Swaine B, Gauthier-Gagnon C. Quality of life of persons with lower-limb amputation during rehabilitation and at 3-month follow-up. Arch Phys Med Rehabil. 2009 Apr;90(4):634-45. doi: 10.1016/j.apmr.2008.11.003. PMID: 19345780.

17. Hafner BJ, Gaunaurd IA, Morgan SJ, Amtmann D, Salem R, Gailey RS. Construct Validity of the Prosthetic Limb Users Survey of Mobility (PLUS-M) in Adults With Lower Limb Amputation. Arch Phys Med Rehabil. 2017 Feb;98(2):277-
285. doi: 10.1016/j.apmr.2016.07.026. Epub 2016 Aug 30. PMID: 27590443; PMCID: PMC5276724.

18. Repo JP, Piitulainen K, Häkkinen A, Roine RP, Kautiainen H, Becker $\mathrm{P}$, Tukiainen EJ. Reliability and validity of the Finnish version of the prosthesis evaluation questionnaire. Disabil Rehabil. $2018 \quad$ Aug;40(17):2081-2087. doi: 10.1080/09638288.2017.1323032. Epub 2017 May 9. PMID: 28486856..

19. Pereira ÂM, Ramos A, Rafaela A, João $M$, Arrifes $V$. Mobility in patients with lower limb amputation after prosthesis. Ann. Med. 2019 Mar 29;51(sup1):212-.

20. Senra H, Oliveira RA, Leal I, Vieira C. Beyond the body image: a qualitative study on how adults experience lower limb amputation. Clin Rehabil. 2012 Feb;26(2):180-91. doi: 10.1177/0269215511410731. Epub 2011 Sep 9. PMID: 21908476.

21. Wurdeman SR, Stevens PM, Campbell JH. Mobility analysis of AmpuTees II: comorbidities and mobility in lower limb prosthesis users. Am J Phys Med Rehabil 2018 Nov;97(11):78

22. Darter BJ, Hawley CE, Armstrong AJ, Avellone L, Wehman $P$. Factors influencing functional outcomes and return-towork after amputation: a review of the literature. J Occup Rehabil. 2018 Dec 1;28(4):656-65

23. La Grow S, Yeung P, Towers A, Alpass F, Stephens C. The impact of mobility on quality of life among older persons. J Aging Health. 2013 Aug;25(5):723-36. doi: 10.1177/0898264313490198. Epub 2013 Jun 3. PMID: 23735305.

24. Wurdeman SR, Stevens PM, Campbell JH. Mobility Analysis of AmpuTees (MAAT I): Quality of life and satisfaction are strongly related to mobility for patients with a lower limb prosthesis. Prosthetics and Orthotics International. 2018;42(5):498-503. doi:10.1177/0309364617736089

25. Shafrin J, Sullivan J, Goldman DP, Gill TM. The association between observed mobility and quality of life in the near elderly. PloS one. 2017 Aug 21;12(8):e0182920.

26. Mussarat Jabeen Khan, Sarah Fatima Dogar, Uzma Masroor family relations, quality of life and post-traumatic stress among amputees and prosthetics. Pak Armed Forces Med J 2018; 68 (1): 125-30

Disclaimer: None to declare.

Conflict of Interest: None to declare.

Funding Sources: None to declare. 